\title{
Stability Range for a Flat Graphene Sheet Subjected to In-Plane Deformation
}

\author{
S. V. Dmitriev ${ }^{a}$, Yu. A. Baimova ${ }^{a}$, A. V. Savin ${ }^{b}$, and Yu. S. Kivshar'c \\ ${ }^{a}$ Institute of Problems of Metal Superplasticity, Russian Academy of Sciences, \\ ul. Khalturina 39, Ufa, 450001 Russia \\ e-mail:julia.a.baimova@gmail.com \\ ${ }^{b}$ Semenov Institute of Chemical Physics, Russian Academy of Sciences, \\ ul. Kosygina 4, Moscow, 119991 Russia \\ ${ }^{c}$ Nonlinear Physics Centre, Australian National University, Canberra, ACT 0200 Australia \\ Received February 3, 2011; in final form, April 21, 2011
}

\begin{abstract}
The effects of the elastic deformation on the mechanical and physical properties of graphene are a subject of intensive current studies. Nevertheless, the stability range for a flat graphene sheet subjected to in-plane deformation is still unknown. Here, this problem is solved by atomistic simulations. In the three-dimensional space corresponding to the $\varepsilon_{x x}, \varepsilon_{y y}$, and $\varepsilon_{x y}$ components of the planar strain tensor, the surface bounding the stability range for a flat graphene sheet has been constructed disregarding the thermal vibrations and the effects of boundary conditions. For the points of this surface, force components $T_{x}, T_{y}$, and $T_{x y}$ have been calculated. It is shown that graphene is structurally stable up to strains on the order of $0.3-0.4$, but it is unstable with respect to the shear in the absence of stretching forces. In addition, graphene cannot preserve its flat shape under the effect of a compressive force since it has zero flexural stiffness.
\end{abstract}

DOI: $10.1134 / \mathrm{S} 0021364011100055$

Graphene is a flat monatomic carbon layer where each atom has covalent bonds with its three neighbors (see Fig. 1a). Due to its unique mechanical, physical, and optical characteristics, graphene is promising for different technological applications. In recent years, the effects of significant elastic strains on the mechanical and physical properties of graphene and other low-dimensional carbon-based structures were widely studied. These studies are in the mainstream of the actively developing technology based on elastic strains [1]. For example, it has been found that the thermal conductivity of graphene and carbon nanotubes decreases gradually with an increase in the tensile stress [2]. The optical conductivity of graphene is also significantly affected by the applied stress [3]. In [4], the effect of uniaxial stretching deformation on the optical atomic vibration modes of graphene was studied by Raman spectroscopy. It was shown that the $G$ and 2 D bands exhibit a significant redshift and the $G$ band becomes split into two bands due to the symmetry lowering induced by the lattice strain. The ab initio calculations demonstrate that the uniaxial stretch strain of up to $10 \%$ does not give rise to the gap in the electron density of states [5], whereas strains can be as high as $03-0.4$ [6]. The experimental estimate for the tensile strength of graphene gives a value of $42 \mathrm{~N} / \mathrm{m}$ [7]. The enhancement of the Young modulus of graphene with the temperature increase from 100 to $500 \mathrm{~K}$ was revealed by the molecular dynamics calcu- lations [8]. The nonlinear theory of elasticity was formulated for graphene in [9], where the ab initio calculations for the constants involved into the defining relationship are also reported. Linear and nonlinear stiffness coefficients were also calculated for graphene through the use of empirical interatomic potentials [10]. Another version of the nonlinear theory of elasticity for graphene was suggested in [11] and was compared to the experimental data within the strain range

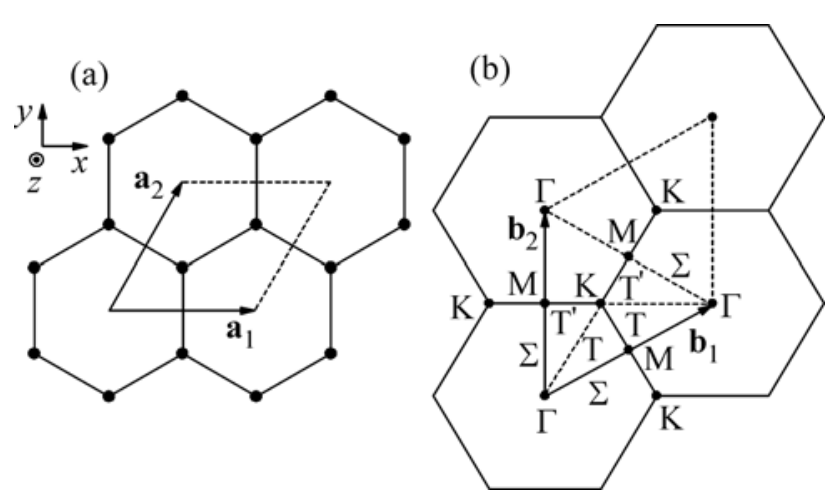

Fig. 1. (a) Crystal lattice of graphene and translation vectors, $\mathbf{a}_{1}$ and $\mathbf{a}_{2}$, for the unit cell containing two carbon atoms. (b) Reciprocal space for the graphene lattice with the first Brillouin zone specified by vectors $\mathbf{b}_{1}$ and $\mathbf{b}_{2}$. Capital Greek letters denote the high-symmetry points and directions in the first Brillouin zone. 
up to $30 \%$. Different causes for the deviations of graphene from the flat shape have been studied: the applied stress [12, 13], effects of substrate [14, 15], and thermal fluctuations [16]. The mica substrate can minimize the deviations of a graphene sheet from the flat shape caused by thermal fluctuations [17]. The electron microscopy of freely suspended graphene sheets reveals the deviations from the flat shape of the order of $1 \mathrm{~nm}$ [18].

The atomic displacement wave arising perpendicular to the graphene sheet can significantly modify its electronic properties [13-15]. In this connection, there appears a task to develop the techniques for controlling the variation of parameters characterizing the transverse atomic displacements in graphene.

The transverse atomic displacements wave was studied by the method of molecular dynamics in the case of uniaxial compression of graphene nanoribbons in the "armchair" directions using the free and supported boundary conditions [12]. The interatomic potential [19] used in [12] leads to a nonzero flexural stiffness of graphene and at supported boundary conditions, buckling arises at a compressive strain of 0.0086 . The periodic transverse displacements appearing in graphene compressed together with the substrate can be used for the selective absorption of hydrogen to modify the electrical properties of graphene [13]. The possibility of periodic transverse displacements in a graphene sheet resulting from the negative thermal expansion was also discussed [14]. In addition, it has been demonstrated that the wavelength and amplitude of the transverse atomic displacement wave in the freely suspended graphene sheer can be controlled due to the internal and thermal stresses [15].

Here, we show that the transverse atomic displacement wave in graphene can also be generated by applying shear strains.

As follows from the aforementioned works, the elastic deformation of graphene is an efficient tool for the modification of its characteristics. Note that the characteristics can be varied in a wide range since we should take into account the three-dimensionality of the space corresponding to planar deformation and the possibility of distorting graphene by a strain of tens percent. In this connection, it is important to determine the theoretical limits for the strain in graphene. Our study is aimed at the calculation of the surface in the space of strains and forces, which confines the stability range for a flat defect-free graphene sheet without taking into account the thermal vibrations and the effects of the boundary conditions.

The graphene unit cell determined by translation vectors $\mathbf{a}_{1}$ and $\mathbf{a}_{2}$ (Fig. 1a) contains two atoms, each having three degrees of freedom, namely, the components of the displacement vector. The $x$ axis coincides with the "zigzag" direction and the $y$ axis coincides with the armchair direction. The reciprocal space for graphene is depicted in Fig. 1b where capital Greek letters denote the high-symmetry points and directions in the first Brillouin zone.

In this work, the instability of the flat graphene lattice is studied with respect to all $3(N-1)+3$ modes of linear vibrations, where $N$ is the number of atoms in the system with periodic boundary conditions. It is convenient to consider separately $3(N-1)$ phonon vibration modes stemming from three modes corresponding to the uniform distortion of the graphene unit cell. The instability criterion with respect to phonon vibration modes is the vanishing of frequency for a certain vibration mode within the first Brillouin zone. The analysis of stability with respect to three modes corresponding to the uniform distortion is reduced to considering the second variation of the potential energy at small increments in the components of the uniform strain. The instability occurs if the matrix composed of the coefficients in the corresponding linear equations loses its positive definiteness.

In this work, this problem is solved by the atomistic simulations using a standard set of interatomic potentials taking into account the energy needed for a distortion of valence bonds, as well as changes in valence and torsion angles. The detailed justification for the choice of the parameters characterizing these potentials is given in [20]. Note that the used set of interatomic potentials was verified by solving a number of problems, for example, in the studies of the thermal conductivity of graphene ribbons with rough edges [20], the thermal conductivity of carbon nanotubes interacting with the substrate [21], the properties of discrete breathers in carbon nanotubes [22], and vibration modes localized at graphene boundaries [23, 24]. Note that other potentials are also used for graphene; in particular, the Brenner potential [19] is quite popular. The characteristic feature of the potential used in [20-24] is that it reproduces the dispersion curves of graphene better than the Brenner potential [21]. Since the stability analysis for the crystal lattice is based on calculations of the dispersion curves, we choose the standard set of potentials used in the molecular dynamics simulations [20]. Let us also mention interesting works $[25,26]$ on the structural modeling of interatomic interactions in graphene.

The standard set of potentials [20] allows us to determine the length of the valence bond $\rho_{0}=1.418 \AA$ for undistorted graphene, for which the equilibrium lattice parameter is $a=\left|\mathbf{a}_{1}\right|=\left|\mathbf{a}_{2}\right|=\sqrt{3} \rho_{0}$.

In graphene, we enumerate atoms by three indices and represent their positions as $\mathbf{r}_{i j k}=i \mathbf{a}_{1}+j \mathbf{a}_{2}+\mathbf{s}_{k}$, where integer numbers $i$ and $j$ determine the number of a unit cell and $k=1,2$ is the number of an atom within the unit cell. The translation vectors can be chosen as $\mathbf{a}_{1}=a(1,0)$ and $\mathbf{a}_{2}=(a / 2)(1, \sqrt{3})$ (see Fig. 1a) and the shear vectors of sublattices are $\mathbf{s}_{1}=0$ 

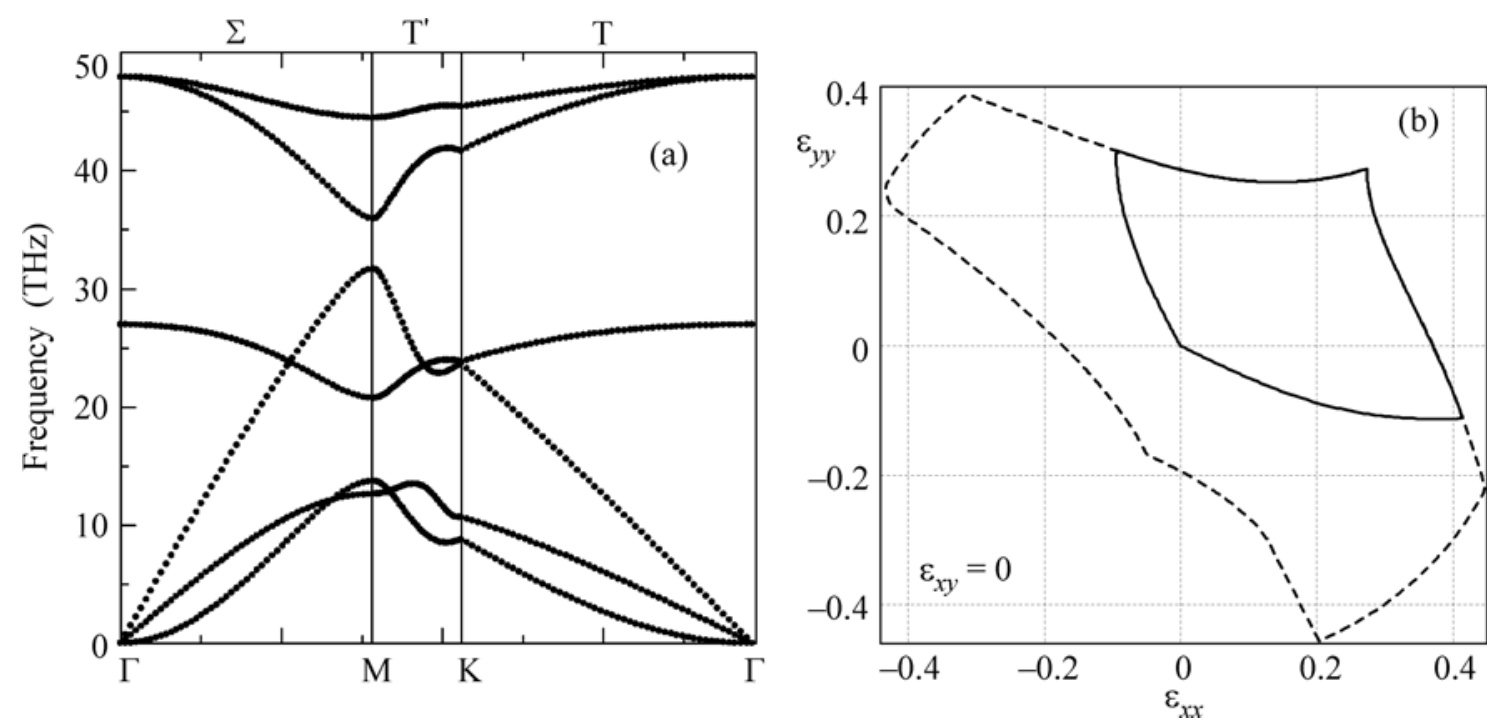

Fig. 2. (a) Dispersion curves for undistorted graphene. (b) The stability ranges for orthotropic graphene under strains $\varepsilon_{x x}=\varepsilon_{y y} \neq 0$ and $\varepsilon_{x y}=0$ determined with respect to (solid curve) phonon vibration modes and (dashed curve) small increments of the uniform strain.

and $\mathbf{s}_{2}=(a / 2)(1,1 / \sqrt{3})$. We subject the graphene lattice to uniform in-plane deformation characterized by three strain components $\varepsilon_{x x}, \varepsilon_{y y}$, and $\varepsilon_{x y}$. For the distorted lattice, the translation vectors should have the form $\mathbf{p}_{1}=\mathbf{a}_{1}+\mathbf{a}_{1} \mathbf{H}$ and $\mathbf{p}_{2}=\mathbf{a}_{2}+\mathbf{a}_{2} \mathbf{H}$, where the $\mathbf{H}$ matrix has the components $h_{11}=\varepsilon_{x x}, h_{12}=h_{21}=\varepsilon_{x y} / 2$, and $h_{22}=\varepsilon_{y y}$. In the uniformly distorted lattice, the first shear vector can always be chosen as $\mathbf{q}_{1}=\mathbf{s}_{1}=0$, whereas the second shear vector should be found by the minimization of the potential energy of the crystal with respect to its components. As the first approximation, we can recommend choosing the vector $\mathbf{q}_{2}^{(1)}=$ $\mathbf{s}_{2}+\mathbf{s}_{2} \mathbf{H}$. Thus, the equilibrium positions of the graphene atoms under the applied strain can be written as $\mathbf{r}_{i j k}=i \mathbf{p}_{1}+j \mathbf{p}_{2}+\mathbf{q}_{k}$. At the next stage, we solved the linear stability problem for this equilibrium atomic array. Analyzing the stability with respect to the phonon vibration modes, we scanned the first Brillouin zone over both coordinates with a step of $0.01 \pi$ and we determined six vibration eigenfrequencies at each point. The crystal was treated as unstable if an eigenfrequency turned out to be imaginary at least one point of the Brillouin zone. As was mentioned above, we also checked the stability of graphene with respect to small increments in three components of the uniform strain. In these calculations, we took into account the work of applied forces related to virtual changes in the size and shape of the graphene unit cell. This corresponds to the assumption that the loadapplying unit is characterized by absolute rigidity. Note that taking into account the finite rigidity of the load-applying unit could somehow reduce the stability range for graphene.
In Fig. 2a, we show the dispersion curves for undistorted graphene at the points in the Brillouin zone located at the $\Sigma, T^{\prime}$, and $T$ segments sequentially connecting the points $\Gamma \longrightarrow \mathrm{M} \longrightarrow \mathrm{K} \longrightarrow \Gamma$ (see Fig. 1b). There are three acoustic and three optical branches. The acoustic branches with the highest and intermediate frequencies correspond to the longitudinal and transverse in-plane atomic vibrations in the graphene sheet. The low-frequency branch corresponds to the transverse out-of-plane atomic vibrations. The waves corresponding to the last branch have zero speed of sound owing to the vanishing flexural stiffness of graphene. Note quite satisfactory agreement of our calculated dispersion curves with those measured by $\mathrm{X}$-ray [27, 28] and Raman [29] scattering techniques, as well as with the dispersion curves calculated based on the empirical potentials [30].

Undistorted graphene is isotropic but the uniform deformation leads, in general, to the loss of isotropy. Only under uniform stretching/compression deformation, $\varepsilon_{x x}=\varepsilon_{y y} \neq 0$, and $\varepsilon_{x y}=0$, graphene remains isotropic, whereas graphene becomes orthotropic at zero shear strain and at $\varepsilon_{x x} \neq \varepsilon_{y y} \neq 0$.

The stability ranges for orthotropic graphene (at $\left.\varepsilon_{x y}=0\right)$ in the $\left(\varepsilon_{x x}, \varepsilon_{y y}\right)$ plane in Fig. 2b. In this figure, the solid curve bounds the stability range with respect to phonon vibration modes and the dashed curve is the boundary of the stability range with respect to small increments of the uniform strain, disregarding the work of applied forces related to the virtual distortion of the graphene unit cell. The second criterion can obviously be disregarded, since it gives the stability range boundary partially coinciding with that following from the first criterion and, in the other part, it 

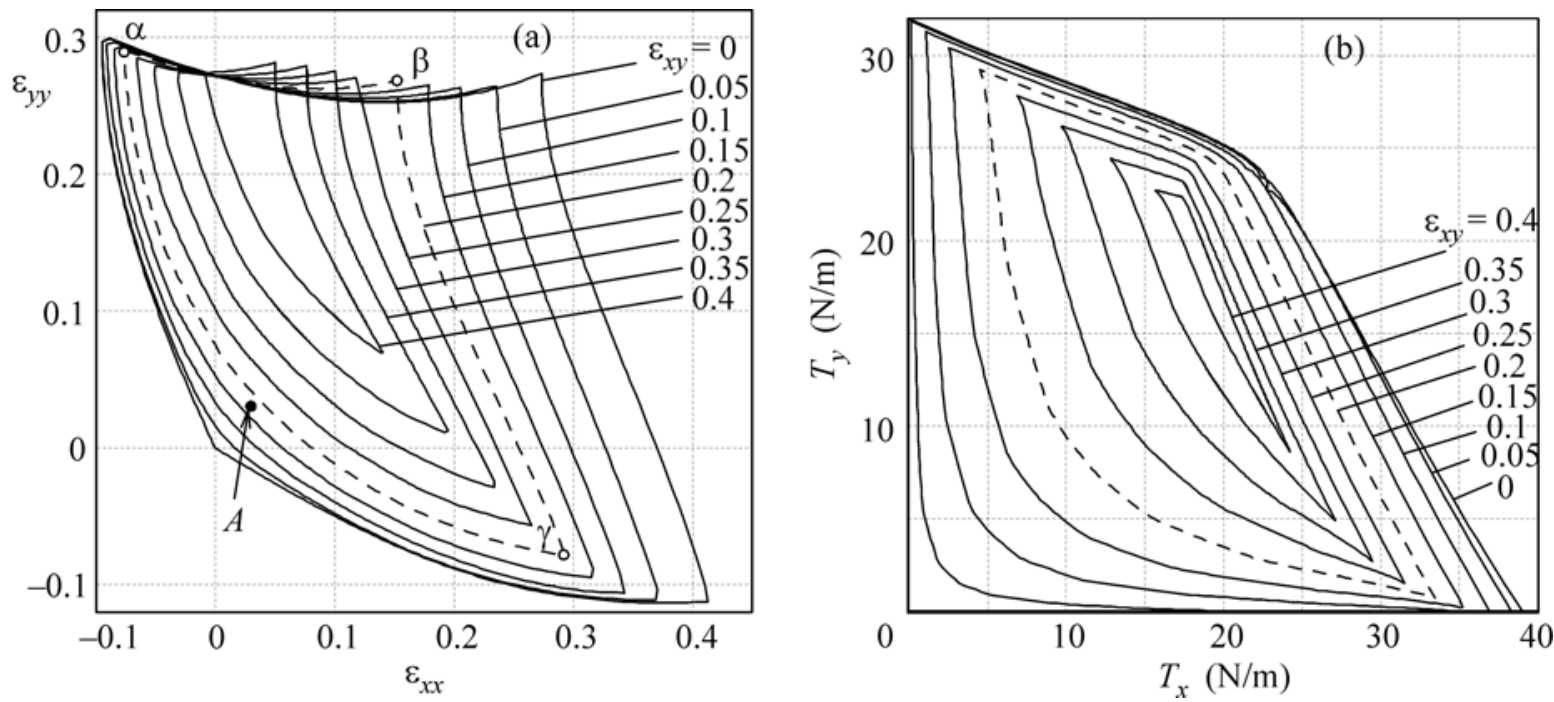

Fig. 3. (a) Surface bounding the stability range for graphene in the three-dimensional strain space represented by its cross sections by the $\varepsilon_{x y}=$ const planes. (b) The $T_{x}$ and $T_{y}$ forces calculated for the curves shown in panel (a).

predicts the higher critical strains in comparison to those following from the first criterion. It turns out that this conclusion remains valid for graphene even in the case of $\varepsilon_{x y} \neq 0$; therefore, we do not address further the results of the stability analysis with respect to increments of the uniform strain. However, note that for a two-dimensional crystal with the Lennard-Jones interatomic interaction, it is necessary to consider both stability criterions [31, 32].

In Fig. 3a, the surface bounding the stability range for graphene in the three-dimensional strain space is illustrated by its cross sections by the $\varepsilon_{x y}=$ const planes. In the chosen coordinate system, the stability range for graphene does not depend on the sign of strain $\varepsilon_{x y}$. The stability range boundary corresponding to the cross section $\varepsilon_{x y}=0.2$, which is discussed below, is shown by the dashed line.

In Fig. 3a, we can see, in particular, that graphene subjected to shear deformation cannot keep its flat shape in the absence of applied $\varepsilon_{x x}$ and $\varepsilon_{y y}$ strains.

There are data on the values of the critical strains for graphene at its uniaxial stretching. The following limiting values for the uniaxial strains in graphene were determined by the methods of quantum mechanics and quantum molecular dynamics: $\varepsilon_{x x} \approx 0.38$ and $\varepsilon_{y y} \approx 0.19$ [33]. The calculations in the framework of the density functional theory (DFT) give the critical values $\varepsilon_{x x} \approx 0.37$ and $\varepsilon_{y y} \approx 0.28$ (see Fig. 3 in [6]) and $\varepsilon_{x x} \approx 0.48$ and $\varepsilon_{y y} \approx 0.30$ (see Fig. 3 in [8]). The molecular dynamics calculations for the graphene nanoribbons give the critical strain value $\varepsilon_{y y} \approx 0.31$ [34]. The nanoindentation of graphene by the atomic force microscope allows obtaining strains up to 0.3 [7].
These values agree well with the results of this work, $\varepsilon_{x x} \approx 0.41$ and $\varepsilon_{y y} \approx 0.30$.

It is interesting to reveal what forces acting in the graphene plane appear at the boundaries of the stability range. In Fig. 3b, we give the values of forces $T_{x}$ and $T_{y}$ for all curves represented in Fig. 3a. If we assume that the graphene sheet has a certain thickness $h$, the forces can be recalculated to stresses, $\sigma=T / h$. It is usually assumed that the thickness of the graphene sheet is equal to the interplanar distance in graphite, $h=3.34 \AA$ [6]. The stability boundary for graphene in the space of forces is situated in the first quadrant, implying that graphene cannot preserve its flat shape under negative (compressive) force components. This is a consequence of zero flexural stiffness of the graphene sheet. The maximum forces withstood by graphene under the uniaxial stretching are $T_{x} \approx$ $39 \mathrm{~N} / \mathrm{m}(117 \mathrm{GPa})$ and $T_{y} \approx 32 \mathrm{~N} / \mathrm{m}(96 \mathrm{GPa})$. The maximum shear force withstood by the flat graphene sheet is about $17 \mathrm{~N} / \mathrm{m}$ (51 GPa); it can be attained only under the effect of stretching forces, $T_{x} \approx 20 \mathrm{~N} / \mathrm{m}$ (60 GPa) and $T_{y} \approx 15 \mathrm{~N} / \mathrm{m}(45 \mathrm{GPa})$.

The determined values of the uniaxial limiting forces can be compared to those reported in other papers. The experiments on the nanoindentation by the atomic force microscope yield the limiting force of $42 \mathrm{~N} / \mathrm{m}$ (126 GPa) [7]. Molecular dynamics gives the limiting force under the uniaxial stretching equal to $T_{x} \approx 58.5 \mathrm{~N} / \mathrm{m}(175 \mathrm{GPa})$ [34] and the quantum mechanical calculations give the following values for the limiting forces: $T_{x} \approx 56 \mathrm{~N} / \mathrm{m}(168 \mathrm{GPa}), T_{y} \approx$ $40 \mathrm{~N} / \mathrm{m}(120 \mathrm{GPa})\left[33\right.$ ] and $T_{x} \approx 40 \mathrm{~N} / \mathrm{m}(120 \mathrm{GPa})$, $T_{y} \approx 38 \mathrm{~N} / \mathrm{m}(115 \mathrm{GPa})$. We can see that the results of this work agree well with the available data. 

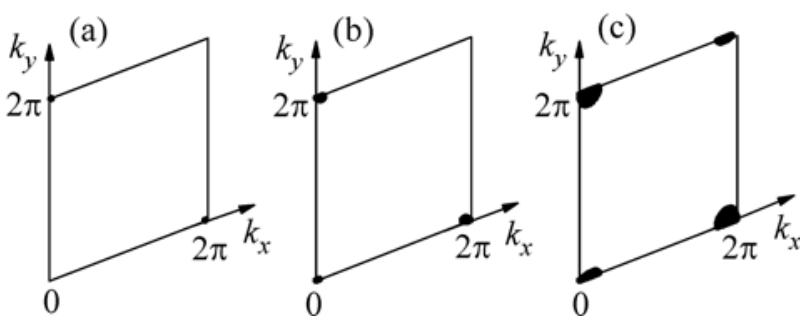

Fig. 4. Evolution of the region with imaginary vibration frequencies in the first Brillouin zone after passing across the boundary surface of the stability range at point $\varepsilon_{x x}=$ $\varepsilon_{y y}=0.03575, \varepsilon_{x y}=0.2$ and the further penetration into the instability range of graphene: (a) $\varepsilon_{x x}=\varepsilon_{y y}=0.035$; (b) $\varepsilon_{x x}=\varepsilon_{y y}=0.03$ (point $A$ in Fig. 3a); (c) $\varepsilon_{x x}=\varepsilon_{y y}=0.01$.

In Fig. 4, we demonstrate an example of the nucleation and evolution of the region with imaginary vibration frequencies (highlighted by black) in the first Brillouin zone after passing across the boundary surface of the stability range and the further penetration into the instability range of graphene. In this case, the shear strain is kept constant, $\varepsilon_{x y}=0.2$, and the components of the stretching strain are kept equal to each other being gradually decreased from $\varepsilon_{x x}=\varepsilon_{y y}=0.03575$ corresponding to the boundary of the stability range down to (a) $\varepsilon_{x x}=\varepsilon_{y y}=0.035$, (b) $\varepsilon_{x x}=\varepsilon_{y y}=0.03$ (point $A$ in Fig. 3a), and (c) $\varepsilon_{x x}=\varepsilon_{y y}=0.01$.

The postcritical behavior of graphene was studied by molecular dynamics for the cell containing $80 \times$ 80 unit cells with the imposed periodic boundary conditions. The equilibrium atomic positions were perturbed by small $\left(10^{-8} \AA\right)$ initial deviations for all three components of the displacement vector. The initial velocities of atoms were assumed to be zero. If atoms exhibited vibrational motion about their equilibrium positions with the amplitude of the order of the initial perturbation, this equilibrium state was considered to be stable. In the case of the instability of the initial uniformly deformed state of graphene, we observed the motion of atoms to the new equilibrium position and further vibrations about it.

The postcritical behavior was analyzed in sufficient detail for the shear strain $\varepsilon_{x y}=0.2$. For this case, the $\alpha \beta \gamma$ curve bounding the stability range is shown in Fig. $3 \mathrm{a}$ as a dashed line. The postcritical behaviors corresponding to the increase and decrease in the strain components $\varepsilon_{x x}$ and $\varepsilon_{y y}$ were qualitatively different. For example, at increasing strain $\varepsilon_{y y}$, the penetration to the instability range across the $\alpha \beta$ boundary was accompanied by the segregation of the valence bonds oriented along the $y$ axis into weak and strong bonds. The crossing of the $\beta \gamma$ boundary gave rise to the segregation of the bonds oriented along the $x$ axis. The most interesting case corresponds to the penetration to the instability range across the $\gamma \alpha$ boundary, which is related to the decrease in the $\varepsilon_{x x}$ and/or $\varepsilon_{y y}$ strains.

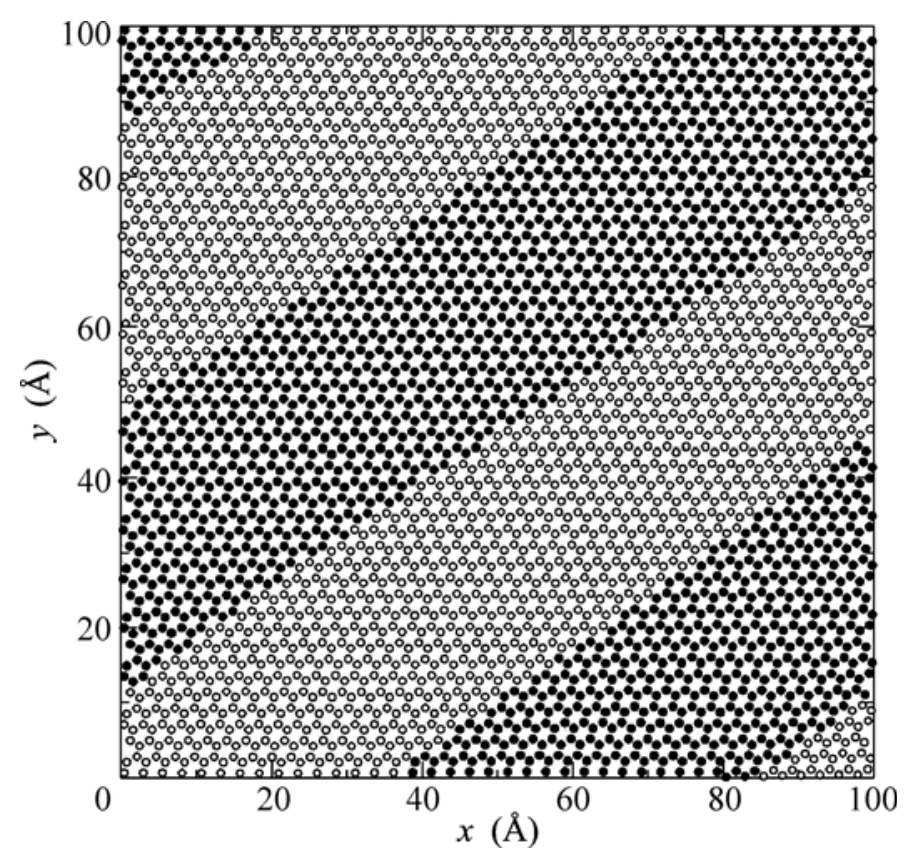

Fig. 5. Graphene structure under strain $\varepsilon_{x x}=\varepsilon_{y y}=0.03$, $\varepsilon_{x y}=0.2$ marked by point $A$ in Fig. 3a. Under this strain, the flat shape of graphene is unstable. The closed and open circles denote the atoms with positive and negative displacements in the $z$ direction, respectively.

Under these conditions, we did not observe the segregation of the valence bonds, and the loss of stability for the flat shape of graphene was attributed to the deviations of atoms in the $z$ direction and the formation of periodic modulations.

An example of the stable graphene configuration with the periodic modulations of the atomic displacements is presented in Fig. 5 for $\varepsilon_{x x}=\varepsilon_{y y}=0.03, \varepsilon_{x y}=$ 0.2 . At this strain, marked by point $A$ in Fig. 3a, the flat shape of graphene is unstable, since point $A$ lies outside the region bounded by the dashed line. The closed and open circles in Fig. 5 denote the atoms with positive and negative displacements in the $z$ direction, respectively. Under specified parameters of the system, the wave of modulated displacements in the direction of the $z$ axis has a nearly sinusoidal shape with an amplitude of $1.8 \AA$ and a wavelength of about $60 \AA$. The atomic displacements in the $x$ and $y$ directions are more than an order of magnitude smaller than those along the $z$ axis. The amplitude of this wave grows with moving away from the stability boundary of the flat graphene shape. The modulation wavelength and the orientation of the wave vector depend on the point in the $\gamma \alpha$ boundary, from which we penetrate into the instability range.

In conclusion, we have calculated the stability boundary for the flat graphene shape under the effect of in-plane strain and analyzed the postcritical behavior of graphene depending on the direction of motion 
in the strain space outside the stability range. If the value of the shear strain is fixed and the penetration into the instability range occurs with an increase in the $\varepsilon_{x x}$ and/or $\varepsilon_{y y}$ strains, we have observed the segregation of the valence bonds into weak and strong ones. If the penetration to the instability range is accompanied by a decrease in the $\varepsilon_{x x}$ and/or $\varepsilon_{y y}$ strains, we have observed the formation of the stable periodically modulated atomic displacements in the direction perpendicular to the graphene sheet.

\section{REFERENCES}

1. T. Zhu and J. Li, Progr. Mater. Sci. 55, 710 (2010).

2. X. Li, K. Maute, M. L. Dunn, and R. Yang, Phys. Rev. B 81, 245318 (2010).

3. F. M. D. Pellegrino, G. G. N. Angilella, and R. Pucci, Phys. Rev. B 81, 035411 (2010).

4. M. Huang, H. Yan, C. Chen, et al., PNAS 106, 7304 (2009).

5. R. M. Ribeiro, V. M. Pereira, N. M. R. Peres, et al., New J. Phys. 11, 115002 (2009).

6. F. Liu, P. Ming, and J. Li, Phys. Rev. B 76, 064120 (2007).

7. C. Lee, X. Wei, J. W. Kysar, and J. Hone, Science 321, 385 (2008).

8. X. Wei, B. Fragneaud, C. A. Marianetti, and J. W. Kysar, Phys. Rev. B 80, 205407 (2009).

9. J.-W. Jiang, J.-S. Wang, and B. Li, Phys. Rev. B 80, 113405 (2009).

10. J.-W. Jiang, J.-S. Wang, and B. Li, Phys. Rev. B 81, 073405 (2010).

11. E. Cadelano, P. L. Palla, S. Giordano, and L. Colombo, Phys. Rev. Lett. 102, 235502 (2009).

12. M. Neek-Amal and F. M. Peeters, Phys. Rev. B 82, 085432 (2010).

13. Z. F. Wang, Y. Zhang, and F. Liu, Phys. Rev. B 83, 041403(R) (2011).

14. R. Miranda and A. L. Vazquez de Parga, Nature Nanotechnol. 4, 549 (2009).

15. W. Bao, F. Miao, Z. Chen, et al., Nature Nanotechnol. 4, 562 (2009).
16. A. Fasolino, J. H. Los, and M. I. Katsnelson, Nature Mater. 6, 858 (2007).

17. C. H. Lui, L. Liu, K. F. Mak, et al., Nature 462, 339 (2009).

18. J. C. Meyer, A. K. Geim, M. I. Katsnelson, et al., Nature 446, 60 (2007).

19. D. W. Brenner, Phys. Rev. B 42, 9458 (1990).

20. A. V. Savin, Yu. S. Kivshar, and B. Hu, Phys. Rev. B 82 , $195422(2010)$.

21. A. V. Savin, B. Hu, and Yu. S. Kivshar, Phys. Rev. B 80, 195423 (2009).

22. A. V. Savin and Yu. S. Kivshar, Eur. Phys. Lett. 82, 66002 (2008).

23. A. V. Savin and Yu. S. Kivshar, Eur. Phys. Lett. 89, 46001 (2010).

24. A. V. Savin and Yu. S. Kivshar, Phys. Rev. B 81, 165418 (2010).

25. A. M. Krivtsov and N. F. Morozov, Phys. Solid State 44, 2260 (2002).

26. I. E. Berinskiy, A. M. Krivtsov, and A. M. Kudarova, in Proceedings of the 36th Summer School on Advanced Problems in Mechanics (St. Petersburg, Russia, 2008), pp. 122-132.

27. J. Maultzsch, S. Reich, C. Thomsen, et al., Phys. Rev. Lett. 92, 075501 (2004).

28. M. Mohr, J. Maultzsch, E. Dobardzic, et al., Phys. Rev. B 76, 035439 (2007).

29. A. Gruneis, R. Saito, T. Kimura, et al., Phys. Rev. B 65, 155405 (2002).

30. L. J. Karssemeijer and A. Fasolino, Surf. Sci. (2010) doi: 10.1016/j.susc.2010.10.036.

31. S. V. Dmitriev, J. Li, N. Yoshikawa, and Y. Shibutani, Phil. Mag. 85, 2177 (2005).

32. S. V. Dmitriev, T. Kitamura, J. Li, et al., Acta Mater. 53, 1215 (2005).

33. Y. Gao and P. Hao, Physica E 41, 1561 (2009).

34. H. Bu, Y. Chen, M. Zou, et al., Phys. Lett. A 373, 3359 (2009).

Translated by K. Kugel 